\title{
Reciprocal Interactions between Neurons and Glia Are Required for Drosophila Peripheral Nervous System Development
}

\author{
Katharine J. Sepp and Vanessa J. Auld \\ Department of Zoology, University of British Columbia, Vancouver, Canada V6T 1Z4
}

\begin{abstract}
A major developmental role of peripheral glia is to mediate sensory axon guidance; however, it is not known whether sensory neurons influence peripheral glial development. To determine whether glia and neurons reciprocally interact during embryonic development, we ablated each cell type by overexpressing the apoptosis gene, grim, and observed the effects on peripheral nervous system (PNS) development. When neurons are ablated, glial defects occur as a secondary effect, and vice versa. Therefore glia and neurons are codependent during embryogenesis. To further explore glial-neuronal interactions, we genetically disrupted glial migration or differentiation and observed the secondary effects on sensory neuron development. Glial migration and ensheathment of PNS axons was blocked by overexpression of activated Rho GTPase, a regulator of actin dynamics. Here, sensory axons extended to the CNS without exhibiting gross pathfinding errors. In contrast, disrupting differentiation by expression of dominant-negative Ras GTPase in glia resulted in major sensory axon pathfinding errors, similar to those seen in glial ablations. Glial overexpression of transgenic components of the epidermal growth factor receptor (EGFR) signaling pathway yielded similar sensory neuron defects and also downregulated the expression of the glial marker Neuroglian. Mutant analysis also suggested that the EGFR ligands Spitz and Vein play roles in peripheral glial development. The observations support a model in which glia express genes necessary for sensory neuron development, and these genes are potentially under the control of the EGFR/Ras signaling pathway.
\end{abstract}

Key words: Grim; EGFR; Spitz; Vein; Pointed; Yan; Neuroglian

\section{Introduction}

Glial cells are important mediators of sensory neuron development in Drosophila (for review, see Auld, 1999; Pielage and Klämbt, 2001). For example, in the peripheral nervous system (PNS), glial cells help guide sensory axons into the CNS (Sepp et al., 2001). Reciprocally, PNS glia require neurons for their development, because glia at later stages use axon tracts to migrate to their final positions along the peripheral nerves (Giangrande, 1994; Reddy and Rodrigues, 1999b; Sepp et al., 2000).

Insight into the signals involved in Drosophila neuron-glia interactions has come from studies in the CNS. Drosophila epidermal growth factor receptor (EGFR) signaling is a key mediator of CNS glial survival (Lanoue et al., 2000; Hidalgo et al., 2001; Bergmann et al., 2002). For example, midline glia survival is dependent on physical contact with commissural neurons and mediated through the EGFR ligand Spitz (Sonnenfeld and Jacobs, 1995; Lanoue et al., 2000; Bergmann et al., 2002). Similarly, lon-

\footnotetext{
Received Dec. 16, 2002; revised June 9, 2003; accepted June 12, 2003.

K.J.S. is supported by a Rick Hansen Neurotrauma Initiative fellowship. This work was supported by grants from the Natural Science and Engineering Research Council of Canada and the Canadian Institutes of Health Research. We sincerely thank Andrea Brand, Ulrike Gaul, Michael Hortsch, Roger Jacobs, Bill Leiserson, Alan Michelson, Marek Mlodzik, John Nambu, Sarb Ner, Hiroki Oda, Norbert Perrimon, Trudi Schüpbach, the Bloomington Stock Center, and the Developmental Studies Hybridoma Bank for antibodies and fly stocks. We also thank Jennifer Bonner and Joost Schulte for helpful comments on this manuscript.

Correspondence should be addressed to Dr. Vanessa Auld, 6270 University of British Columbia, Vancouver, British Columbia, Canada V6T 1Z4. E-mail: auld@zoology.ubc.ca.

Copyright $\odot 2003$ Society for Neuroscience $\quad$ 0270-6474/03/238221-10\$15.00/0
}

gitudinal glia in the CNS require EGF receptor signaling through the Neuregulin-like ligand Vein for survival (Hidalgo et al., 2001). Conversely, glia help maintain CNS neuronal survival (Booth et al., 2000), and later in larval stages CNS glia have also been shown to control the proliferation of neuroblasts (Ebens et al., 1993).

The glia of the PNS in Drosophila have a significant number of differences from their CNS counterparts. Peripheral glia migrate over substantially larger distances than CNS glia. They are also molecularly distinct because they express proteins not expressed by CNS glia, such as Gliotactin (Auld et al., 1995). Furthermore, they do not undergo any natural cell death during embryogenesis (Sepp et al., 2000). Given these differences, do peripheral glia and neurons reciprocally interact to promote long distance cell migration, and how might this occur?

Both axon misguidance and sensory neuronal loss can be caused by ablation of peripheral glia or disruption of early glial differentiation (Reddy and Rodrigues, 1999a; Sepp et al., 2001). Thus sensory neurons depend on glial cells for major aspects of their development. Reciprocally, it is not known whether glial cells in Drosophila require sensory neurons for their development. In zebrafish embryos, however, it has recently been shown that PNS glia use the lateral line axon tracts as an essential migratory substrate (Gilmour et al., 2002). In mutants in which the lateral line axon tracts were misplaced, the glial cells would be misrouted as a secondary consequence (Gilmour et al., 2002). It is 
not known, however, whether peripheral glia are capable of migrating in the complete absence of neurons.

Here, we performed neuronal ablations to determine whether sensory neurons contribute to PNS glial development. We found that peripheral glial development is indeed dependent on sensory neurons. Interestingly, the embryonic phenotypes of neuronal ablation mirrored the effects of glial ablation, suggesting that a reciprocal interaction between PNS neurons and glia occurs. PNS glia-neuron interactions were further explored by disrupting Rho GTPase and EGFR/Ras signaling pathways. It was found that EGFR signaling mediates the differentiation of peripheral glia and potentially regulates the expression molecules involved in glia-neuron cross talk.

\section{Materials and Methods}

Fly strains and genetics. For glial and neuronal ablation, the repo-GAL4 (Sepp et al., 2001) and elav-GAL4 lines (Bloomington Stock Center) were crossed to a UAS-grim line (Wing et al., 2001) to generate repo::grim and elav::grim progeny, respectively. For ectopic expression of the GTPases presented in Figure 4, the repoGAL4 line carrying a UAS-Actin-GFP marker (Verkhusha et al., 1999) was crossed to UASRhoV14 (Fanto et al., 2000) and UAS-Ras1N17 (Lee et al., 1996) to generate repo::RhoV14 and repo::Ras1N17 progeny, respectively. For Ras pathway signaling analysis, repo-GAL4 flies were crossed to UAS-Ras1N17 (Lee et al., 1996), $U A S-\operatorname{Ras}^{A C T}$ (Lu et al., 1993), UAS-Egfr ${ }^{D N}$ (O'Keefe et al., 1997), UAS-lambdaEGFR (Queenan et al., 1997), and UAS-Yan ${ }^{A C T}$ (Rebay and Rubin, 1995) to generate repo::RasN17, repo::Ras $1^{A C T}$, repo::Egfr ${ }^{D N}$, repo::lambdaEGFR, and repo::Yan ${ }^{A C T}$ progeny, respectively. EGFR pathway loss-of-function mutants were generated previously: $E g f r^{f^{2}}$ ( $f l b^{1 K 35}$ ) (Schejter and Shilo, 1989), spitz ${ }^{1}\left(\right.$ spi $^{A 14}$ ) (Nüsslein-Volhard et al., 1984), pointed $\Delta^{88}$ (Scholz et al., 1993), and vein ${ }^{L 6}$ (Simcox et al., 1996). Oregon R was the wild type. Embryos were raised at $25^{\circ} \mathrm{C}$ except for those carrying the actin-green fluorescent protein (GFP) marker, which were raised at $21^{\circ} \mathrm{C}$.

Immunohistochemistry. Embryos were stained as reported previously (Halter et al., 1995). Primary antibody concentrations were as follows: mouse monoclonal antibody (mAb) 22C10/anti-Futsch (Developmental Studies Hybridoma Bank, University of Iowa, Iowa City, IA) at 1:2, mouse mAb 1B7 (Hall and Bieber, 1997) at 1:500, rabbit anti-Repo at 1:200 (Halter et al., 1995), rabbit anti-Gliotactin at 1:400, rabbit anti- $\beta$ galactosidase (Cappel, Aurora, OH) at 1:500, rabbit anti-GFP (AbCAM, Cambridge, UK) at 1:200, mouse anti-diphosphoERK (Sigma) at 1:300, and rabbit anti-HRP (Jackson ImmunoResearch, West Grove, PA) at 1:100. HRP reactions were done using the Vectastain Elite ABC kit (Vector Laboratories, Burlingame, CA) according to manufacturer's instructions. The goat anti-rabbit alkaline phosphatase-conjugated secondary (Jackson ImmunoResearch) was used at 1:600. For immunofluorescence, the goat antimouse Alexa 568 and goat anti-rabbit Alexa 488 antibodies were used (Molecular Probes, Eugene, OR), and embryos were mounted in Vectashield (Vector Laboratories). Images were collected on a Bio-Rad Radiance Plus laser scanning confocal microscope. Confocal stacks were projected using Image 1.24 . Chemically labeled embryos were mounted in $90 \%$ glycerol in PBS and imaged on a Zeiss Axioskop fitted with a Sony Power HAD 3CCD color video camera. All digital images were compiled using Adobe Photoshop 5.5.
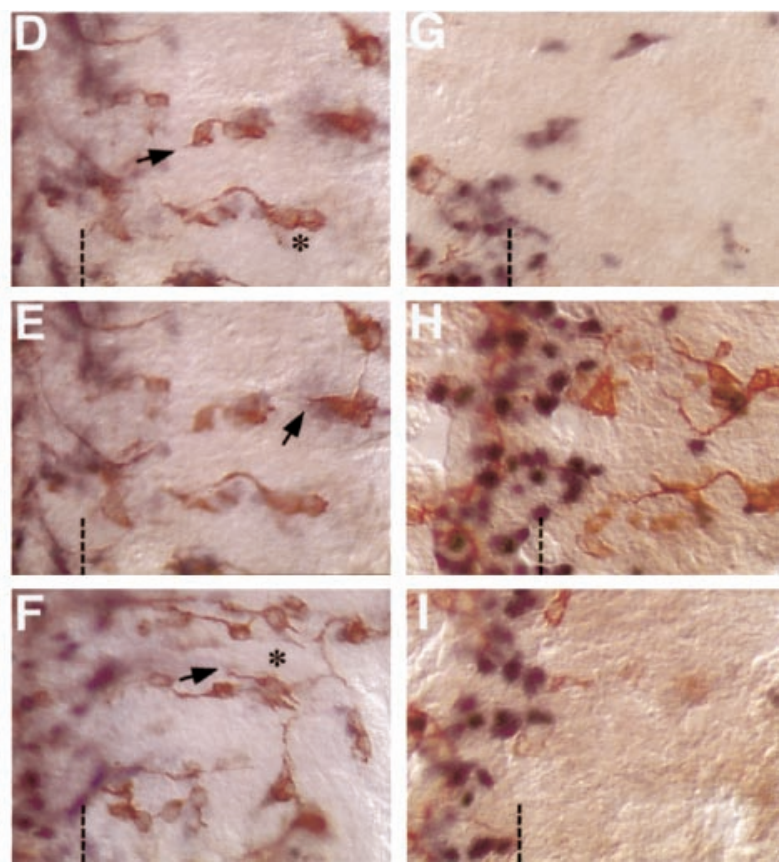

Figure 1. Early ablation of neurons causes loss of glia and vice versa in the peripheral nervous system. For glial nuclear labeling, embryos were stained with anti-Repo (blue). Neurons were counterstained with anti-Futsch (brown). Anterior is to the top, (ventral) is off to the left, and dorsal is on the right. The border between the CNS and periphery is marked with a dashed CNS and a (arowheads). C, A repo..ghim embrowhere glia are targeted for ablation. Glial nucleiremain at the lateraledge in little or no Repo-positive glial cells being present in the affected segments of the peripheral nervous system. The presence of more sensory axons $(H)$ correlated with a greater incidence of Repo-positive glia being present.

\section{Results}

\section{Ablation of neurons in early development disrupts glia and vice versa}

To determine whether peripheral glial cells require neurons during embryonic development, ablations of neurons and glia were performed. To ablate neurons, the elav-GAL4 line was used to drive expression of the apoptosis gene construct, UAS-grim (Wing et al., 2001). The elav-GAL4 driver is expressed exclusively in all post-mitotic embryonic neurons (and not glia). The grim gene blocks the activity of Drosophila inhibitor of apoptosis protein (DIAP1) gene, which is required to prevent caspase activation causing apoptosis (Goyal et al., 2000). The activity of the GAL4/UAS system (Brand and Perrimon, 1993) is heterogeneous from cell to cell (Lee and Luo, 1999; Sepp et al., 2001); thus the efficacy of cell death induction varied across different hemisegments (Fig. $1 B$ ). Ablation of neurons during embryogenesis had striking secondary effects on glial development. In elav::grim embryonic segments with the strongest ablation of neurons, there was a corresponding loss of Repo-positive glial cells (Fig. $1 A, B$, compare arrows). Some glial nuclei with weak Repo immunoreactivity relative to other glia in less severely ablated segments were also observed (Fig. 1G). As was expected, the heterogeneity in timing of cell ablation resulted in a mosaic, and thus we were unable to perform a quantitative analysis of these effects. Of all neuronal-ablated hemisegments, however, 69\% showed some 
degree of loss of Repo-positive glia $(n=236)$ compared with controls (Fig. 1G-I). This percentage is likely to be an underrepresentation because glial numbers in many hemisegments were ambiguous as a result of the disorganization and clumping of glial nuclei and were not scored. The greatest loss of Repopositive glia correlated with those segments that had the greatest degree of neuronal ablation (Fig. 1, compare $G, I, H$ ). The absence of Repo-positive glia was not caused by migration of the peripheral glia into other regions of the nervous system or other focal planes because there was no corresponding increase in glia in the neighboring regions (Fig. 1I). The data suggest that peripheral glia require the presence of neurons for their development during embryogenesis.

Next, glial ablation was compared with neuronal ablation. Previous analysis has shown that peripheral glial ablation causes sensory axon guidance defects (Sepp et al., 2001); however, loss of Futsch-positive neurons was not characterized. All PNS glia were targeted for ablation using the repo-GAL4 driver to activate $U A S$ grim (repo::grim). The repo-GAL4 line is specifically expressed in all post-mitotic PNS glia as well as subsets of CNS glia during embryogenesis stages 12-17 (Sepp et al., 2001). The repo-GAL4 line shows no activity in neurons. In embryos with strong glial ablation, only faded anti-Repo staining within the few remaining glial nuclei was observed (Fig. $1 C$, arrowheads), similar to those seen in neuron-ablated embryos (Fig. $1 B$, arrowheads). In the severely glial-ablated embryos, a striking loss of Futsch-positive sensory neuronal cell bodies in the PNS was observed (Fig. 1C-F, asterisk). Again given the mosaic nature of the GAL4 system, a detailed quantitation of the phenotypes was not possible, but of all hemisegments in which glia were ablated, 77\% showed some degree of loss of the Futsch-positive sensory neurons $(n=153)$. The absence of Futsch-positive neurons was not caused by movement from their usual positions because no evidence of the neuronal cell bodies could be detected through multiple focal planes (Fig. 1D,E). The absence of one or more of the chordotonal sensory organs was seen frequently (Fig. $1 D, F$, asterisk), and those sensory neurons that were present often had shortened or misrouted axons (Fig. 1D-F, arrows). Therefore, sensory neurons require the presence of glial cells for their proper development during embryogenesis.

\section{Late neuronal and glial cell death: effects on PNS patterning}

The next aim was to determine whether reciprocal interactions of PNS neurons and glia occur during their migration-axon extension phases (stages 13-15). Embryos in which neurons were triggered into apoptosis during later development using elav::grim expression were analyzed. At the later stages, sensory neurons from the ventral, lateral, and dorsal clusters have arisen and extend axons over varying distances depending on their age. The late-apoptotic neuronal phenotypes were compared with lateapoptotic glial phenotypes in repo::grim embryos (stages 13-15). In elav::grim embryos in which many sensory neurons had survived to mid-neurodevelopment, we noticed severe axon pathfinding defects. Many axons appeared stalled (Fig. $2 B$, solid arrows), and some neurons showed no axogenesis at all (Fig. $2 B, D$, concave arrowheads). Sensory axons also showed defects in the direction of their extension. Although in all hemisegments sensory axons always projected in an overall correct ventral direction, many sensory neurons strayed significantly off course (Fig. $2 B$, concave arrow). Some sensory axons misrouted such that they traversed over to their neighboring hemisegments (Fig. 2D, concave arrow). Therefore, it appears that sensory neurons un-
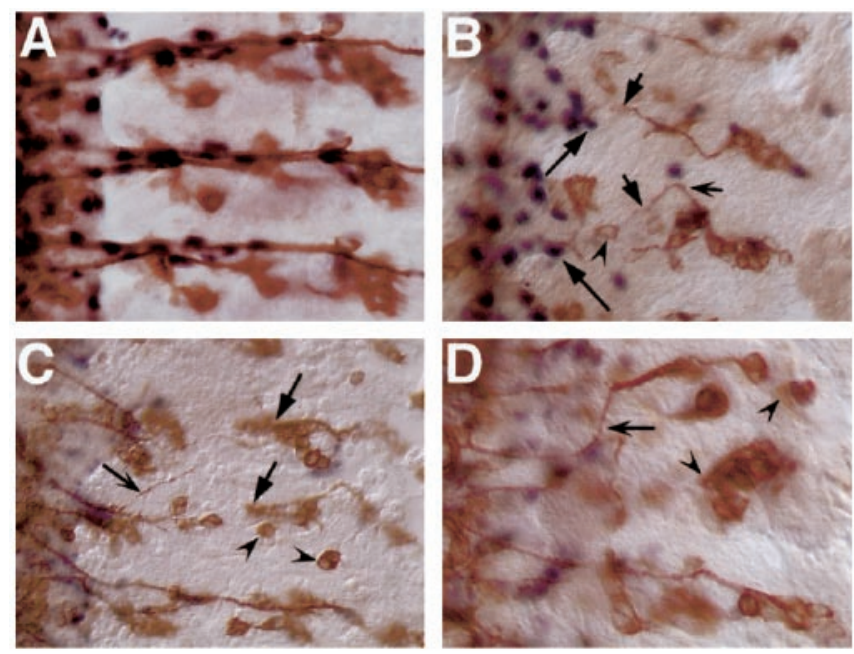

Figure 2. Late death of glia and neurons: effects on pathfinding. For glial nuclear labeling, embryos were stained with anti-Repo (blue). Neurons were counterstained with anti-Futsch (brown). Anterior is to the top, and midline (ventral) is off to the left. $A$, A wild-type stage 16 embryo. Neuron-ablated elav::grim embryos $(B, D)$ and a glial-ablated repo::grim embryo $(C)$ show similar sensory axon extension and pathfinding defects. They include axon stalls (solid arrows), total failure of axogenesis (concave arrowheads), and gross misguidance errors that include axons pathfinding across segment boundaries (concave arrows). $B$, In cases in which no axons traverse the CNS-PNS transition zone in the ventral region, peripheral glial nuclei appear stalled in their birthplace (long arrows).

dergoing cell death during their axon extension phase have significant axogenesis and misguidance phenotypes.

The phenotypes of late neuronal-ablated embryos are remarkably similar to those of late glial-ablated embryos, which have been described in detail previously (Sepp et al., 2001). Briefly, in glial-ablated repo::grim embryos, sensory neurons show axonal stalling (Fig. 2C, solid arrows), misrouting across segmental boundaries (Fig. 2C, concave arrows), as well as absolute failure of axogenesis (Fig. 2C, concave arrowheads).

\section{Physical contact requirements for glial and sensory axon migration}

Normally, peripheral glia migrate into the periphery in contact with axons (Sepp et al., 2000). Because peripheral glia have never been observed to migrate without axonal contact, it has been hypothesized that they require axons as migrational substrates (Sepp et al., 2000). By examining neuron-ablated embryos, the hypothesis could be investigated further. In elav::grim embryos, in which no axons connected between the dorsolateral region of the embryo to the CNS, peripheral glia remained within the ventral CNS-PNS transition zone (TZ) (Figs. $1 B, 2 B$, longer stemmed arrows). The observations support the hypothesis that peripheral glia require axon tracts for migration.

We next investigated whether sensory axons require direct physical contact with glia as they extend centrally. To do this, peripheral glia were prevented from extending cytoplasmic processes by expressing a constitutively activated form of the Rhol GTPase (UAS-RhoV14) using the repo-GAL4 driver. Rho GTPase is involved in actin dynamics of migrating cells in many different tissues (for review, see Settleman, 2001). Overexpression of RhoV14 in glia prevented peripheral extension of cell processes (Fig. 3C,D, glia in green, arrows). Furthermore, glial cell bodies remained stalled in the CNS-PNS transition zone. The distances between the glia and all sensory neuron birthplaces were many times greater than the length of growth cones or their filopodial 

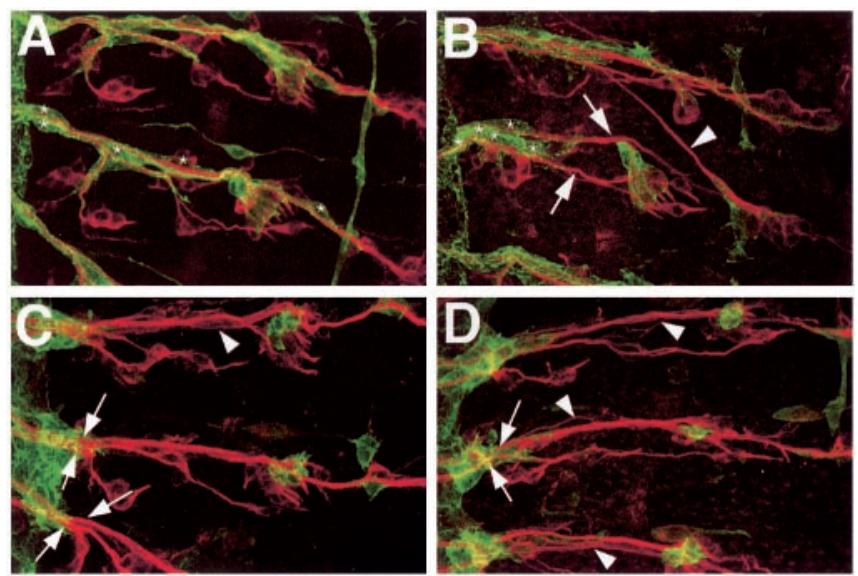

Figure 3. Continuous contact of glial sheath by growth cones is not required for sensory axon migration. For glial staining, embryos expressing glial actin-GFP were labeled with anti-GFP (green). Sensory neurons were counterstained with anti-Futsch (red). Anterior is at the top, and midline is to the left. $A$, A wild-type stage 16 embryo shows complete glial ensheathment of sensory neuronal tracts. The stereotypic position of the glia nuclei, which can be detected by their characteristic shape and lack of actin-GFP, are shown (asterisks). B, repo::Ras1N17 embryo shows incomplete peripheral glial ensheathment of nerves (arrows) as well as sensory axons crossed incorrectly to the neighboring anterior segment (arrowhead). Glia nuclei are clustered at the CNS-PNS boundary (asterisks) C, D, Two examples of repo::RhoV14 embryos are shown, in which glial extension of cytoplasmic processes is blocked. Sensory axon pathways to the CNS are normal and organized into two tracts (arrows) as in wild-type hemisegments. The tracts are defasciculated (arrowheads), which is likely caused by the loss of the glial sheath.

reach. This phenotype was observed in every hemisegment analyzed ( $n=196)$. Surprisingly, sensory axon pathfinding defects such as axons misrouting past intersegmental boundaries, or loss of Futsch-positive neurons, were never observed, in contrast to glialablated embryos. The only noticeable sensory axonal tract defect was defasciculation (Fig. 3C,D, arrowhead), which was most likely caused by the absence of glial sheaths and is not a pathfinding error. Therefore, sensory axonal projection to the CNS is not dependent on continuous contact with the peripheral glia.

\section{Disruption of glial Ras signaling leads to sensory axon migration defects}

Currently there are few candidate molecules that could act as mediators of neuron-glia interactions in the PNS; however, evidence from the Drosophila CNS suggests that neuron-glia interactions occur through a number of receptor tyrosine kinases (RTKs) expressed by midline and longitudinal glia (Klämbt et al., 1992; Sonnenfeld and Jacobs, 1994; Scholz et al., 1997; Shishido et al., 1997; Hidalgo et al., 2001). Ras GTPase is activated by RTKs, and it has pleiotropic functions in the nervous system, including differentiation (Scholz et al., 1997) and trophic survival of CNS glia (Bergmann et al., 2002).

To determine whether Ras1 signaling in peripheral glia mediates aspects of PNS glial interaction with sensory neurons, a dominant-negative variant of Ras1 (UAS-Ras1N17) was overexpressed in glia using the repo-GAL4 driver. As with overexpression of UAS-grim, the magnitude of effects that were observed in each hemisegment was variable. In repo::RasN17 embryos, glia were stalled (asterisks) but could extend cytoplasmic processes to a greater extent along the peripheral axons compared with repo::RhoV14 mutants (Fig. 3, compare $B, C, D$ ). Strikingly, sensory axons in the repo::RasN17 embryos showed pathfinding defects more similar to glial-ablated repo::grim embryos than to repo::RhoV14 embryos (compare Figs. $2 B, 3 B$ ). Sensory axons strayed well away from their normal direct trajectories to the CNS, and neuronal cell bodies were misplaced. There was also a variable loss of HRP-positive sensory neurons. Sensory axon pathfinding defects (not counting simple defasciculation) in repo::RasN17 embryos occurred in $23.4 \%$ of all hemisegments $(n=166)$, compared with none in repo::Actin-GFP wild-type controls $(n=108)$. Because altered Ras1 signaling in glia causes sensory axon migration defects, it is possible that Ras1 signaling affects the regulation of molecules important for glia-neuron interactions in the PNS.

\section{Downregulation of EGFR signals in glia affects PNS neuronal development}

Given the results above, the next aim was to test a candidate signaling pathway that could be involved in neuron-glia interaction via Ras1 signaling. Because Ras can be activated through different receptors, it was important to determine what receptors mediate peripheral glial Ras signaling. In other invertebrate glia, fibroblast growth factor receptors (FGFRs) mediate glial morphogenesis (Klämbt et al., 1992; Condron, 1999); however, neither of the two known FGFRs, Breathless and Heartless, has peripheral glial expression (Klämbt et al., 1992; Shishido et al., 1997). On the other hand, the EGFR is broadly expressed throughout the embryo and also activates Ras1. Specificity of its activity is regulated through multiple ligands and modulating factors that are expressed in the neurons and glia of the PNS (Forjanic et al., 1997; Lanoue et al., 2000).

To test whether EGFR tyrosine kinase signaling occurs endogenously within peripheral glia, we assayed peripheral glia for the presence of activated mitogen-activated protein (MAP) kinase. Antibodies to the diphosphorylated extracellular signal-related kinase (diphosphoERK) protein have been used previously to map cells that contain activated MAP kinase and thus reflect receptor tyrosine kinase activity during development (Gabay et al., 1997a,b). We found that peripheral glia were positive for diphosphoERK, and thus receptor tyrosine kinase signaling pathway occurs in these cells during their development (Fig. 4). Activated MAP kinase was seen in the peripheral glia as they migrate (Fig. $4 B, C$ ) and persisted to later stages (Fig. $4 D$ ). In addition, activated MAP kinase was detected in the glia at the lateral edge of the CNS including the intersegmental glia and the exit glia (Fig. 4E).

To test the function of EGFR signaling in peripheral glia, a dominant-negative form of the EGFR (repo::Egfr ${ }^{D N}$ ) was overexpressed in glia, and the resultant effects on PNS development were observed. The phenotypes were compared with overexpression of the dominant-negative form of its downstream target, Ras1 (repo::Ras1N17), and overexpression of activated Yan (repo::Yan ${ }^{A C T}$ ). Yan is an ETS transcription factor that is inactivated by EGFR signaling and is an inhibitor of differentiation in many cell types, including glia (Rebay and Rubin, 1995; Gabay et al., 1996). It was expected that PNS glial downregulation of EGFR and Ras1, as well as overactivation of Yan, should generate similar nervous system phenotypes if EGFR signaling is important for PNS glial development.

As noted earlier, the activity of the GAL4/UAS system that was used to drive expression of the transgenic constructs was heterogeneous and generated a range of phenotypic severity between embryonic hemisegments as well as individual embryos. The penetrance and severity of the phenotypes increased depending on what temperature the embryos were raised at because GAL4 activity is increased at higher temperatures. Overall, the classes of phenotypes observed in repo::Egfr ${ }^{D N}$, repo::Ras1N17, and repo::Yan ${ }^{A C T}$ mutants were similar. PNS patterning defects in 

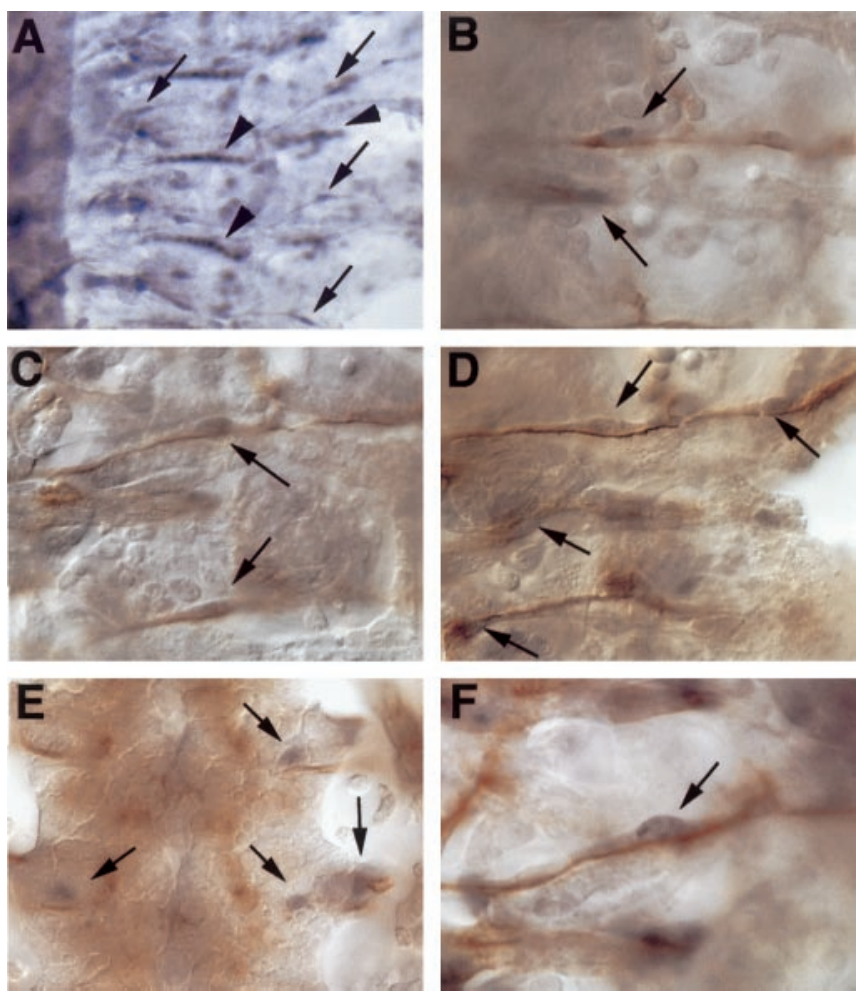

Figure 4. Activated MAP kinase in peripheral glia during embryonic development. Embryos were labeled with anti-diphosphoERK (black) in all panels and costained with anti-fasciclin II (brown) $B-F$, Anterior is to the top, and CNS is to the left in all panels except $E$, where it is centered. $A$, Activated MAP kinase is detected in the nuclei of peripheral glia (arrows) along with the strong staining in the muscle attachment sites (arrowheads). $B-D$, The nuclei of peripheral glia are associated with labeled neurons (brown) and are positive for activated MAP kinases (black).E, At the lateral edges of the CNS, the intersegmental glia and exit glia are also positive for activated MAP kinase (arrows) as are the longitudinal glia (not shown in this focal plane). $F$, High magnification of a peripheral glia nucleus. $A, 200 \times$ magnification; $B-E, 630 \times$ magnification; F, $1000 \times$ magnification.

embryonic hemisegments were observed at 52.1\% $(n=186)$, $47.8 \%(n=205)$, and $37.4 \%(n=163)$, respectively, at $25^{\circ} \mathrm{C}$. In moderately affected hemisegments, sensory axon pathfinding errors were observed as well as incorrect fasciculation-bundling of nerves in the CNS-PNS transition zone (Fig. 5C, $F, G$ ). In severely affected embryos, sensory axon pathfinding was highly erratic, and sensory neuron cell bodies were misplaced (Fig. $5 E, F, H$ ). In some embryos, there was a clear loss of HRP-positive sensory neurons (Fig. $5 D, H$ ), but in general this particular phenotype was difficult to assess because of the overall disorganization of the PNS (Fig. 5F). Motor neuron patterning was also disrupted in severely affected embryos (Fig. $5 D, F, H$ ). Interestingly, glial expression of activated EGFR or activated Ras1 (repo::lambdaEgfr and repo::Ras $1^{A C T}$, respectively) did not cause any disruption of either peripheral glial or neuronal development compared with wild-type embryos (data not shown). Together, the data show that downregulation of EGFR signaling in glia causes secondary effects on neuronal development in the PNS.

\section{Glial EGFR signals regulate Neuroglian expression}

To determine whether the downregulation of EGFR signaling in glia disrupts glial differentiation, the expression of Neuroglian (Nrg) in glia expressing EGFR pathway mutants was monitored. $\mathrm{Nrg}$ is an L1-like Ig cell-adhesion molecule (Bieber et al., 1989) that is expressed by both PNS neurons and glia during embryonic neurodevelopment. Two isoforms of Nrg are expressed in the embryo. The mAb 1B7, which recognizes both isoforms, stains neurons, glia, epidermis, somatic musculature, tracheae, and gut (Hall and Bieber, 1997). Nrg is an appropriate choice as a readout for EGFR-mediated glial differentiation, because it is expressed strongly in peripheral glia only in their mature axon ensheathment phase during late embryogenesis (Hall and Bieber, 1997; our unpublished data).

To determine whether Nrg is downstream of EGFR signaling, the differential intensity of mAb 1B7 Nrg antibody labeling in peripheral glia versus the epidermis was analyzed in repo::Egfr ${ }^{D N}$, repo::Ras1N17, and repo::Yan ${ }^{A C T}$ mutants using confocal microscopy. Confocal sections for each mutant were collected as one large stack, including the epidermis and the PNS, and then later separated to compare expression of Nrg in the nervous system compared with the epidermis alone. In the wild type, expression of Nrg in both the peripheral glia and epidermis is robust (Fig. $6 A, B$, Nrg in green, HRP neuronal staining in red). In contrast, expression of Nrg in the PNS glia is reduced compared with epidermal $\mathrm{Nrg}$ levels in repo::Egfr${ }^{D N}$, repo::Ras1N17, and repo::Yan ${ }^{A C T}$ mutants (Fig. $6 \mathrm{C}-\mathrm{H}$ ). Sensory neuronal Nrg staining could be observed in most cases (Fig. 6C, E, G, concave arrows). As with the previous experiments, penetrance and severity of this phenotype increased with higher temperatures. It is unlikely that the weakened Nrg staining is caused simply by an absolute loss of peripheral glia, because the prevailing glial phenotype in these mutants was stalling (Fig. 3B). If Nrg expression had been maintained, we would have expected concentrated $\mathrm{Nrg}$ staining in the CNS-PNS transition zone region (Fig. 6C, E, G, solid arrows) of the mutant embryos. Thus the data suggest that Nrg expression could be regulated by EGFR signals in peripheral glia and that EGFR signaling does mediate peripheral glial differentiation.

\section{EGFR signaling regulates both PNS glial and neuronal development}

To confirm that EGFR signaling plays an integral role in PNS development, loss-of-function mutations in EGFR pathway genes were analyzed. These included EGFR and its ligands Spitz (a TGF- $\alpha$ homolog) and Vein (a Neuregulin-like protein) and its downstream effector Pointed (an ETS transcription factor) (for review, see Perrimon and Perkins, 1997). All of these genes belong to the "spitz group," which is involved in common developmental processes such as neurogenesis. For example, in the PNS, Egfr, spitz (spi), and pointed (pnt) mutants have been shown previously to lack at least two of the five lateral chordotonal sensory organs in each hemisegment (Fig. $7 E, H, N$, solid arrowheads) (Rutledge et al., 1992; Okabe and Okano, 1997; Rusten et al., 2001). In the current study, it was found that vein ${ }^{L 6}(\mathrm{vn})$ homozygotes have a similar loss of lateral chordotonal neurons (Fig. 7J, arrowhead). It was also noted that the Egfr ${ }^{F 2}$ mutant had significant loss of Futsch-positive ventral cluster sensory neurons in all hemisegments and disorganization of the neurons that were present (Fig. $7 B, E$, compare asterisks). The sensory neurons of the EGFR pathway mutants showed other abnormal phenotypes that have not been characterized previously. In $s p i^{1}$ homozygotes, dorsal cluster sensory axons projected incorrectly across segment boundaries (Fig. $7 H$, arrows) in $16 \%$ of hemisegments $(n=88)$. In $v n$ mutants, there was evidence of sensory axogenesis defectsgrowth cone stalling (Fig. $7 K$, solid arrow). As well, in both $v n$ and pnt $\Delta^{88}$ mutants, anterior and posterior sensory axon fascicles were spaced farther apart at the CNS-PNS TZ compared with the wild type (Fig. $7 B, K, N$, compare concave arrows). Abnormal separation of axon bundles in the TZ is associated with aberrant 
or absent peripheral glial wrapping (Sepp et al., 2001; Sepp and Auld, 2003). The data show that EGFR signaling is important for sensory neuronal development not only during neurogenesis but also for the later axogenesis and growth cone pathfinding phase.

To further examine the importance of EGFR function in peripheral glia, the profile of Gliotactin expression was also analyzed in the selected mutants. Gliotactin is a late marker of glial differentiation (Fig. $7 C$, arrow, arrowheads) and is also expressed in the epidermis. There was no detectable expression of Gliotactin in the glia of Egfr, $v n$, and pnt homozygotes, although Gliotactin could be detected in the epidermis (Fig. $7 C, F, L, O$ ). Interestingly, in $s p i^{1}$ mutants, Gliotactin expression was robust in the glia, although the glial morphological profiles in spi mutants were not entirely normal. In cases in which dorsal cluster sensory axons had migrated incorrectly, the dorsolateral peripheral glia migrated along the aberrant axonal pathways (Fig. $7 I$, arrows). Abnormalities in the peripheral glial sheaths were also observed in regions in which there were no apparent sensory axon defects (Fig. 7I, solid arrowhead). Overall, the peripheral glia were essentially able to extend cytoplasmic processes in $s p i^{1}$ mutants, and ventral peripheral glia were able to separate from the peripheral glia of the main axon tracts as in the wild type (Fig. 7C,I, compare concave arrowheads). Together with the transgene overexpression studies, the loss-offunction mutant data suggest that EGFR signaling is important for both glial and neuronal development in the PNS. Furthermore, the data support the hypothesis that EGFR signaling is potentially a mediator of PNS glia-neuron interactions.

\section{Discussion}

Through ablation studies, we have shown that reciprocal interactions occur between glia and sensory neurons. It was thought previously that peripheral glia guide sensory axon migration by acting as a passivepermissive migrational substrate. Surprisingly, blocking glial sheath extension by activated Rho does not significantly alter sensory axon pathfinding, but expression of dominant-negative Ras1 does. There-

fore, glia are not simply passive substrates for axon extension. The observation that EGFR pathway downregulation in glia causes similar phenotypes to downregulation of Ras 1 activity suggests that the EGFR pathway could be a key mechanism in glial development. This hypothesis was supported by the analysis of EGFR loss-of-function mutations. EGFR signaling in PNS glia is likely to be pleiotropic and could affect multiple aspects of PNS development, as discussed below.
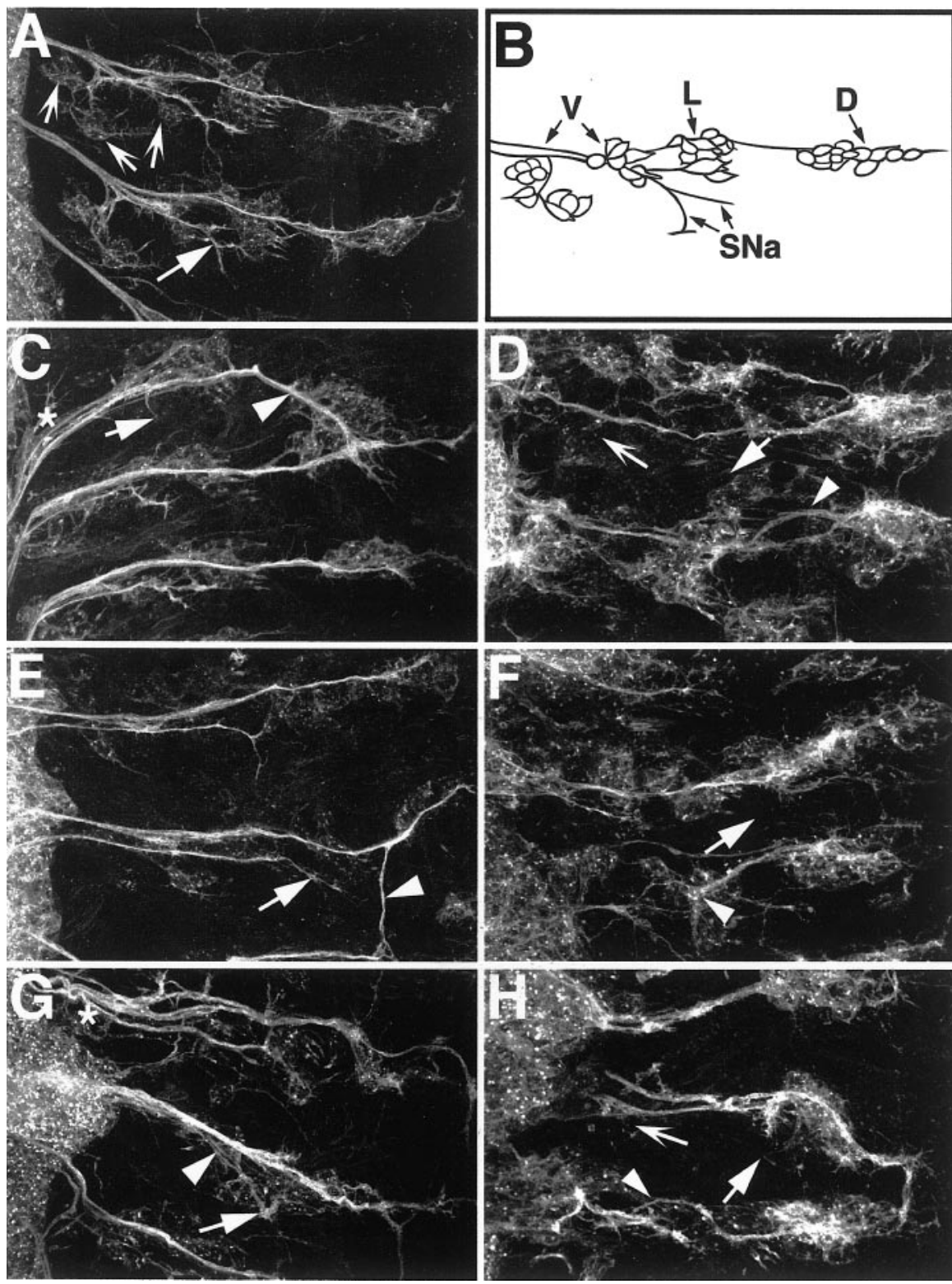

Figure 5. Downregulation of EGFR signals in glia causes neuronal defects. For sensory and motor neuronal staining, embryos were labeled with anti-HRP. Anterior is to the top, and CNS is to the left. $A$, A mature wild-type embryo. The segmental nerve a (SNa) motor branch (solid arrow) and the ventral cluster sensory neurons (concave arrows) are indicated. B, A schematic diagram of sensory neurons and motor neurons that are labeled by the anti-HRP antibody. Sensory neurons are organized into ventral (V), lateral (L), and dorsal (D) clusters, and their cell bodies project axons to the CNS. Motor neurons whosecell bodies are not shown project axons from the CNS to the somatic musculature in the periphery. The SNa motor branch is indicated. For simplicity, the other motor branches of the PNS are not shown. In the ventral region, motor and sensory axons are bundled together into combined fascicles by peripheral glia. C, D, repo:: Egfr ${ }^{D N}$ embryos. E, F, repo:: Ras $1 N 17$ embryos. G, $H$, repo::Y Yan ${ }^{A C T}$ embryos. All mutant embryos show similar classes of phenotypes, and the genotypes cannot be distinguished from each other if they are observed "blind." The PNS patterns in all mutants ( $(-H)$ do not match the wild type $(A)$. Sensory neuron cell bodies are generally misplaced, and their axons show frequent misrouting and stalls ( $C-H$, arrowheads). The SNa motor axon branches often have abnormal projections or are missing ( $(-H$, solid arrows). Fasciculation of sensory-motor bundles is abnormal $(C, G$ asterisks). There is a loss of sensory neurons in the ventral regions of severely affected embryos ( $D, H$, concave arrows).

\section{Glial cells and neuronal axogenesis}

When glial cells are ablated during early stages of neuronal growth, axons project at incorrect trajectories and show frequent stalling. Thus it is possible that PNS glia assist sensory neuronal axogenesis from early stages. This hypothesis is supported further by previous observations that PNS glia appear to stimulate the expression of Futsch, a microtubule-associated protein involved in axon extension (Klaes et al., 1994; Hummel et al., 2000). Also, 

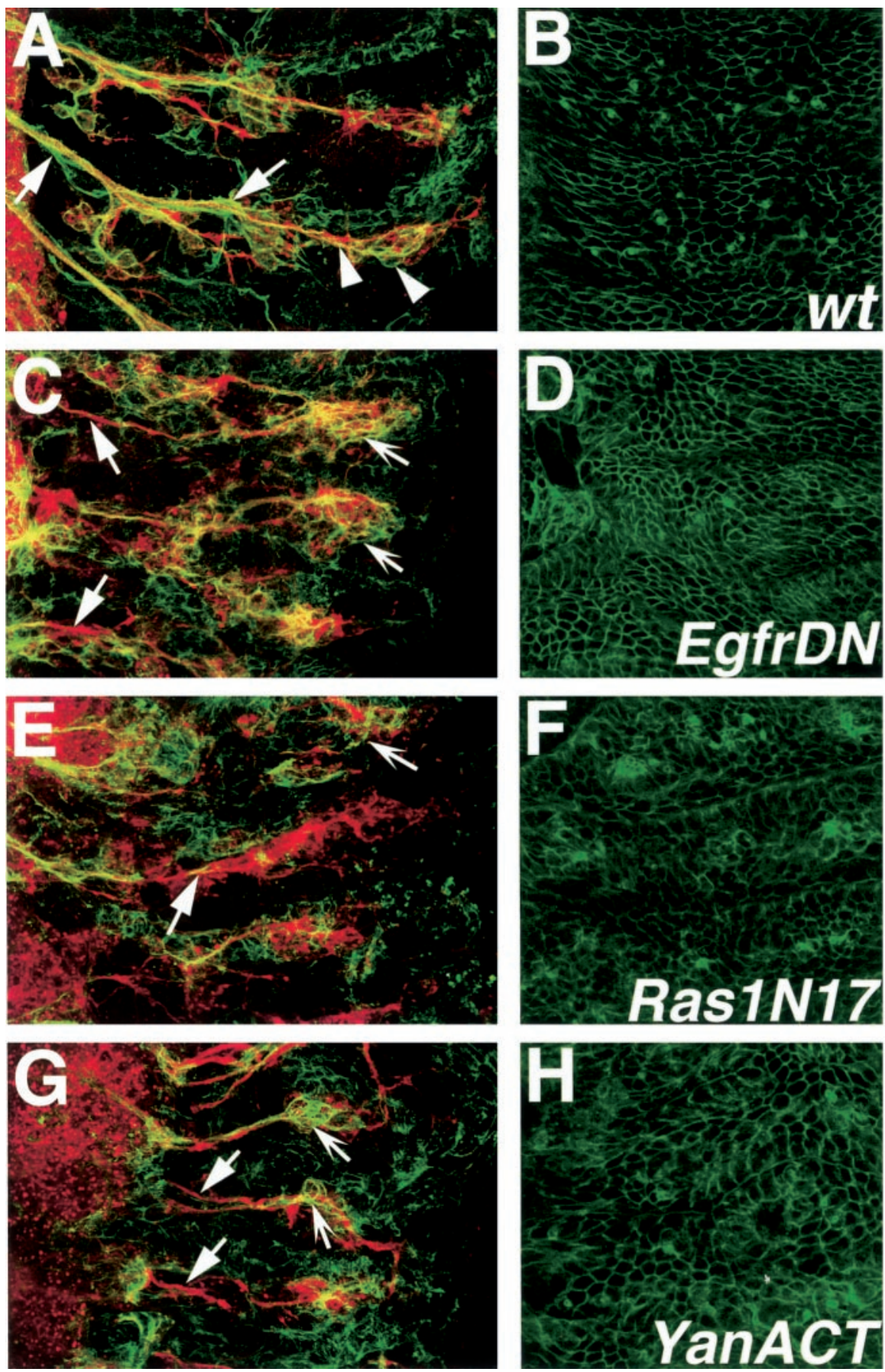

Figure 6. Glial Neuroglian expression is downregulated in EGFR pathway mutants. Embryos were labeled with mAb $1 B 7$ (anti-Nrg, green) and anti-HRP (red). Confocal stacks of wild-type and mutant embryos were split and projected such that the nervous system and the epidermis are shown on the left- and right-hand columns, respectively. $A$, Wild-type mature embryo shows peripheral glial expression of $\mathrm{Nrg}$ (solid arrows) in a profile similar to that in Figure $3 A$. Nrg is also expressed by sensory neurons (arrowheads). $B$, The epidermis of wild-type embryos has strong Nrg staining around the circumference of the cells. $C, D$, repo::Egfr ${ }^{D N}$ embryo. $E, F$, repo::Ras $1 N 17$ embryo. $G, H$, repo:: Yan ${ }^{A C T}$ embryo. $C, E, G$, Peripheral glial Nrg expression in mutant embryos is weak (solid arrows); however, sensory neuronal Nrg staining can be detected (concave arrows). D, F, H, The epidermal expression level of $\mathrm{Nrg}$ is not visibly downregulated.

from analysis of the fly wing and sensory macrochaete on the notum, glia appear to guide the initial projection of sensory axons (Giangrande, 1994; Manning and Doe, 1999; Reddy and Rodrigues, 1999a,b). Thus, glial guidance of sensory projections could be a general role for the growth of many different sensory structures.
What could the nature of a glialderived signal that promotes axogenesis be? It is possible that peripheral glia could act as either (1) a passive substrate for axon migration or (2) a source of molecules that attract and facilitate entry of sensory axons into the CNS. In support of the first possibility, during wild-type PNS development, sensory axons extend through a sleeve of peripheral glia sheaths (Sepp et al., 2000). This could represent a situation in which the glia simply prevent neuronal growth cones from migrating on incorrect substrates. This hypothesis was tested by blocking the extension of glial sheaths by ectopic expression of active Rho GTPase. Because sensory axons migrated correctly, constant glial contact is not necessary for axon pathfinding. Thus it is more likely that peripheral glia provide a secreted cue(s) to guide sensory axons. The analysis of dominant-negative Ras1 expression in glia suggests that expression of the secreted cue could be transcriptionally controlled through Ras1 signaling. As well, the cue could serve a dual function as a trophic factor. In support of this was the reduction of Futsch-positive neurons and the pathfinding defects in repo::Ras1N17 mutants, which were qualitatively similar to elav::grim neuron-ablated mutants.

\section{Ras signaling in glia and the EGFR pathway}

As alluded to earlier, Ras signaling has pleiotropic effects, including cell proliferation, survival, and differentiation (for review, see Perrimon and Perkins, 1997). Therefore, interpretation of Ras signaling downregulation is complex because effects of altered cell differentiation versus disrupted cell viability might not be separable. Potentially this is why the phenotypic effects observed in repo::EGFR ${ }^{D N}$, repo::Ras1N17, and repo::Yan ${ }^{A C T}$ embryos were diverse.

The EGF receptor is the first receptor tyrosine kinase identified that has a major role in peripheral glial development. EGFR is broadly expressed, and tissuespecific effects of EGFR activation are generated by the binding of different ligands and modulators (Perrimon and Perkins, 1997). Peripheral glia have strong expression of Star (Forjanic et al., 1997, their Fig. $5 L$ ), which modifies the activity of the EGFR ligands Spitz (Forjanic et al., 1997) and Keren (Reich and Shilo, 2002). Also, the EGFR downstream effector, Pointed P1, is expressed strongly in peripheral glia (Klämbt, 1993). The EGFR ligand Vein is related to vertebrate Neuregulin and is expressed by a number of CNS neurons that extend axons into the PNS, including the anterior corner cell (aCC), RP2, and the VUMs (Lanoue et al., 2000; Hidalgo et al., 
2001). Of interest to this work is that aCC contacts the peripheral glia during its early migration phase at a time when it is positive for Vein mRNA expression (Sepp et al., 2000). Thus aCC or other Veinexpressing neurons could act as a potential source of a ligand for EGF receptor signaling in the peripheral glia, the majority of which arise at the lateral edge of the CNS. We have shown that activated MAP kinase is present in peripheral glia, and given that the peripheral glia have the necessary components to be regulated by EGFR, this suggests that EGF receptor signaling controls peripheral glia development.

Further support for EGFR signaling during peripheral glia development comes from our observation that downregulation of EGFR signals in the glia disrupted their differentiation as assayed by expression of Neuroglian and Gliotactin, two proteins known to be expressed in terminally differentiated glia (Auld et al., 1995; Hall and Bieber, 1997). For instance, loss-offunction mutants in the EGF receptor (either dominant-negative EGFR or Egfr hypomorphic alleles) clearly resulted in a lack of Gliotactin expression. Similarly, pointed and vein mutants also resulted in a lack of Gliotactin expression. These results suggest that the EGF receptor and its ligand Vein play a critical role in the differentiation of peripheral glia. On the other hand, loss-of-function mutants in Spitz did not block Gliotactin expression. The peripheral glia were able to extend processes and separate from the main axon tract; however, the glial morphological profiles in spitz mutants were not completely normal, suggesting that Spitz plays a role albeit more minor than Vein during PNS glia differentiation. Conversely the defects in the peripheral glia in Spitz mutants could reflect an underlying defect in the sensory neurons. Overactivation of EGFR signals in PNS glia by expression of activated EGFR or activated Ras1 did not significantly alter peripheral glia development. This is possibly caused by mechanisms intrinsic to the EGFR pathway that auto-downregulate EGFR signals, such as EGFR-dependent expression of its negative regulator, Argos (Golembo et al., 1996; Jin et al., 2000).

The question still remains whether EGFR signaling is necessary for glial survival or simply just for the final differentiation steps. For these studies we were not able to determine whether the peripheral glia undergo apoptosis when EGFR signaling is disrupted because detection of apoptotic cells using terminal deoxynucleotidyl transferase-mediated biotinylated UTP nick end labeling or single-stranded DNA detection was inconclusive. In general, the normal complement of five to six peripheral glial nuclei could be seen clustered at the PNS-CNS after repo::rasN17 and repo::EGFR ${ }^{D N}$ expression (Fig. 3, compare $A, B$ ), but given the mosaic nature of GAL4 expression, it was impossible to accu-
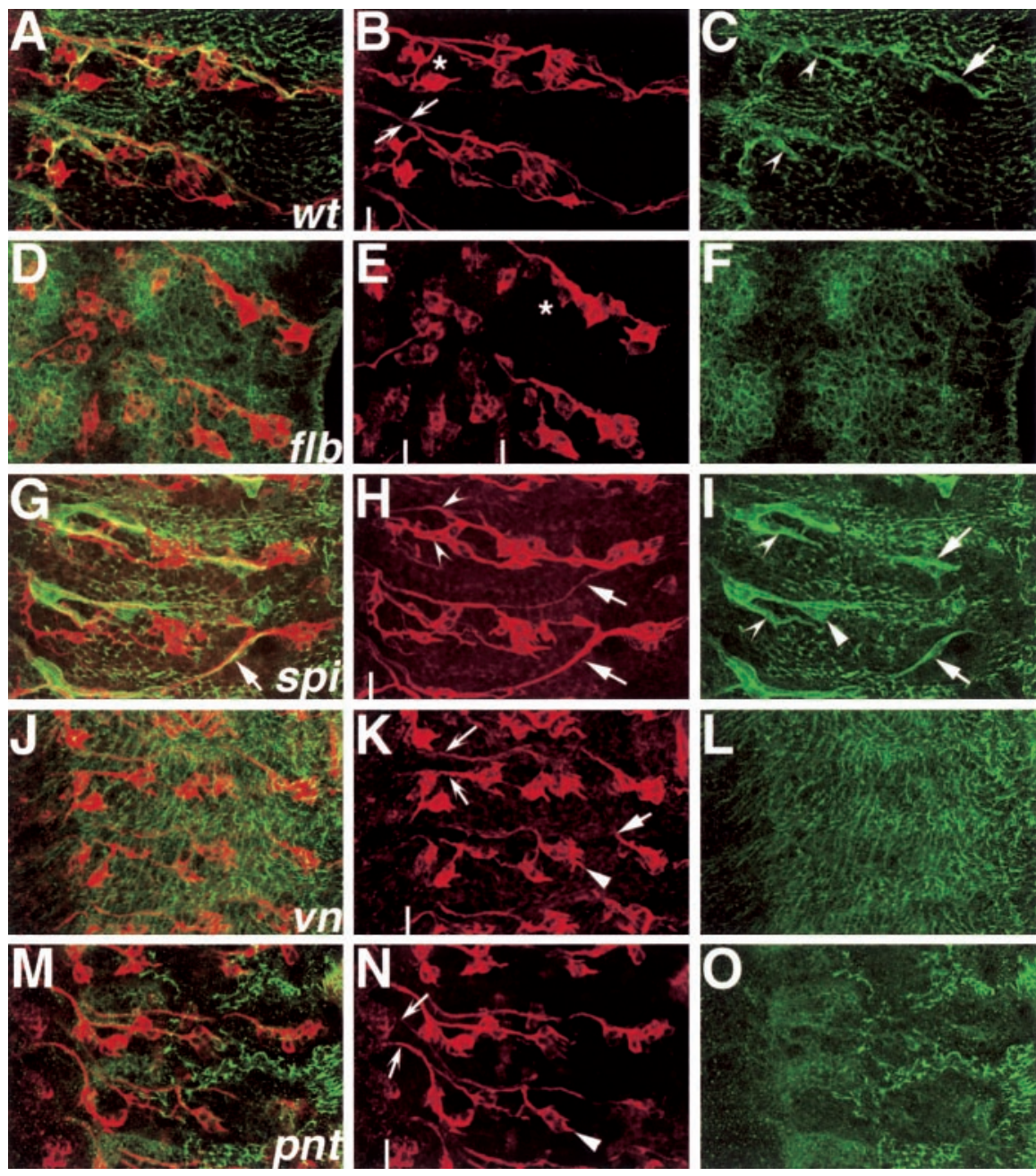

Figure 7. EGFR pathway loss-of-function mutants have neuronal and glial PNS defects. Embryos were labeled with anti-Futsch (red) and anti-Gliotactin (green), and PNS hemisegments were imaged with confocal microscopy. Anterior is to the top, andCNS ventral cluster sensory neurons. C, Peripheral glial anti-Gliotactin staining (arrows) overlies punctate epidermal expression. $D-F$ coted $(F)$. G-H, The spi homozygote shows aberrant sensory axon pathfinding across segment

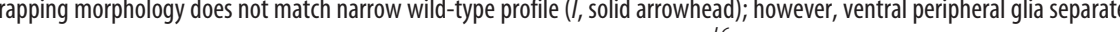
correctly from main nerve trunk (concave arrowheads; compare I, C). J-L, The $v n^{L 6}$ homozygotes show axonal stalling $(K$, solid arrow), and peripheral glial Gliotactin expression is not detected ( $L$ ). $M-0$, The pnt $\Delta^{88}$ homozygotes have disrupted Gliotactin solid arrowheads). Abnormal separation of anterior and posterior peripheral nerve fascicles was noted in spi, vn, and pnt mutants as compared with the wild type ( $B, H, K, N$, concave arrows).

rately quantitate any differences in number of peripheral glia. We have shown previously, however, that the numbers of peripheral glia remain constant through embryonic and larval stages (Sepp et al., 2000) (unlike the midline glia, for example), which suggests that cell death is not normally a mechanism to trim down the numbers of "excess" glia in the PNS.

Of interest in these studies were the secondary effects that disrupting the EGF receptor signaling pathway in the peripheral glia had on the development of the sensory neurons. The disruption in sensory neurons ranged from defects in axongenesis and guidance to the reduction in Futsch-positive sensory neurons and cell bodies. These effects were qualitatively similar to those seen when we drove apoptosis in neurons themselves. We have shown in the current study that vein homozygotes have a similar 
loss of lateral chordotonal neurons as has been reported previously for Egfr, spitz, and pointed mutants (Rutledge et al., 1992; Okabe and Okano, 1997; Rusten et al., 2001). These defects in the loss-of-function mutants could be caused by the effects of EGF receptor signaling during sensory neuron determination or conversely could reflect the underlying defects in the differentiation of the peripheral glia. In support of this possibility, many of the phenotypes seen in the loss-of-function mutants were the same as when EGF receptor signaling was specifically disrupted in the peripheral glia. Phenotypes such as sensory axon projecting across segment boundaries, sensory axogenesis defects-growth cone stalling, and abnormal separation of axon bundles are associated with aberrant or absent peripheral glial wrapping (Sepp et al., 2001; Sepp and Auld, 2003). The data show that EGFR signaling is important for sensory neuronal development not only during neurogenesis, but also for the later axogenesis and growth cone pathfinding phase, and that signaling in the peripheral glia plays an important part in sensory neuron development.

EGFR signaling is likely to be only one of numerous means of PNS glia-neuron interaction. Recently, it has been found that Decapentaplegic- and Hedgehog-mediated signals are responsible for neuron-glia interaction in the Drosophila visual system, and nitric oxide signaling mediates neuron-glia interaction in the Manduca olfactory system (Gibson et al., 2001; Rangarajan et al., 2001). It would be interesting to know whether these mechanisms also have a role in Drosophila PNS development. In conclusion, we have shown that reciprocal interactions between PNS glia and sensory neurons occur during neurodevelopment and that the EGF receptor plays a role in mediating part of these interactions. Our studies mirror those from vertebrates, which have shown that Neuregulins provided by neurons control the development of peripheral glia (Schwann cells) through signaling of the ErbB2 and ErbB3 receptors. In return, Schwann cells provide both neurotrophic factors for neuronal survival and nerve fasciculation (for review, see Garratt et al., 2000). Because Drosophila genetics has been a very powerful means of characterizing evolutionarily conserved molecules involved in axon guidance, there is great potential for knowledge gained from further studies of neuron-glia interaction studies in Drosophila to transfer to other phyla as well.

\section{References}

Auld VJ (1999) Glia as mediators of growth cone guidance: studies from insect nervous systems. Cell Mol Life Sci 55:1377-1385.

Auld VJ, Fetter RD, Broadie K, Goodman CS (1995) Gliotactin, a novel transmembrane protein on peripheral glia, is required to form the bloodnerve barrier in Drosophila. Cell 81:757-767.

Bergmann A, Tugentman M, Shilo B-Z, Steller H (2002) Regulation of cell number by MAPK-dependent control of apoptosis: a mechanism for trophic survival signaling. Dev Cell 2:159-170.

Bieber AJ, Snow PM, Hortsch M, Patel NH, Jacobs JR, Traquina ZR, Schilling J, Goodman CS (1989) Drosophila Neuroglian: a member of the immunoglobulin superfamily with extensive homology to the vertebrate neural adhesion molecule L1. Cell 59:447-460.

Booth GE, Kinrade EFV, Hidalgo A (2000) Glia maintain follower neuron survival during Drosophila CNS development. Development 127:237-244.

Brand AH, Perrimon N (1993) Targeted gene expression as a means of altering cell fates and generating dominant phenotypes. Development 118:401-415.

Condron B (1999) Spatially discrete FGF-mediated signaling directs glial morphogenesis. Development 126:4635-4641.

Ebens AJ, Garren H, Cheyette BNR, Zipursky SL (1993) The Drosophila anachronism locus: a glycoprotein secreted by glia inhibits neuroblast proliferation. Cell 74:15-27.

Fanto M, Weber U, Strutt DI, Mlodzik M (2000) Nuclear signaling by Rac and Rho GTPases is required in the establishment of epithelial planar polarity in the Drosophila eye. Curr Biol 10:979-988.

Forjanic JP, Chen C-K, Jäckle H, Gaitàn MG (1997) Genetic analysis of stomatogastric nervous system development in Drosophila using enhancer trap lines. Dev Biol 186:139-154.

Gabay L, Scholz H, Golembo M, Klaes A, Shilo B-Z, Klämbt C (1996) EGF receptor signaling induces pointed $P 1$ transcription and inactivates Yan protein in the Drosophila embryonic ventral ectoderm. Development 122:3355-3362.

Gabay L, Seger R, Shilo B-Z (1997a) In situ activation pattern of Drosophila EGF receptor pathway during development. Science 277:1103-1106.

Gabay L, Seger R, Shilo B-Z (1997b) MAP kinase in situ activation atlas during Drosophila embryogenesis. Development 124:3535-3541.

Garratt AN, Britsch S, Birchmeier C (2000) Neuregulin, a factor with many functions in the life of a Schwann cell. BioEssays 22:987-996.

Giangrande A (1994) Glia in the fly wing are clonally related to epithelial cells and use the nerve as a pathway for migration. Development 120:523-534.

Gibson NJ, Rössler W, Nighorn AJ, Oland LA, Hildebrand JG, Tolbert LP (2001) Neuron-glia communication via nitric oxide is essential in establishing antennal-lobe structure in Manduca sexta. Dev Biol 240:326-339.

Gilmour DT, Maishchein H-M, Nüsslein-Volhard C (2002) Migration and function of a glial subtype in the vertebrate peripheral nervous system. Neuron 34:577-588.

Golembo M, Schweitzer R, Freeman M, Shilo BZ (1996) Argos transcription is induced by the Drosophila EGF receptor pathway to form an inhibitory feedback loop. Development 122:223-230.

Goyal L, McCall K, Agapite J, Hartwieg E, Steller H (2000) Induction of apoptosis by Drosophila reaper, hid and grim through inhibition of IAP function. EMBO J 19:589-597.

Hall SG, Bieber AJ (1997) Mutations in the Drosophila Neuroglian cell adhesion molecule affect motor neuron pathfinding and peripheral nervous system pathfinding. J Neurobiol 32:325-340.

Halter DA, Urban J, Rickert C, Ner SS, Ito K, Travers AA, Technau GM (1995) The homeobox gene repo is required for the differentiation and maintenance of glia in the embryonic nervous system of Drosophila melanogaster. Development 121:317-332.

Hidalgo A, Kinrade EFV, Georgiou M (2001) The Drosophila Neuregulin Vein maintains glial survival during axon guidance in the CNS. Dev Cell 1:679-690.

Hummel T, Krukkert K, Roos J, Davis G, Klämbt C (2000) Drosophila Futsch/ $22 \mathrm{C} 10$ is a MAP1B-like protein required for dendritic and axonal development. Neuron 26:357-370.

Jin MH, Sawamoto K, Ito M, Okano H (2000) The interaction between the Drosophila secreted protein argos and the epidermal growth factor receptor inhibits dimerization of the receptor and binding of secreted spitz to the receptor. Mol Cell Biol 20:2098-2107.

Klaes A, Menne T, Stollewerk A, Scholz H, Klämbt C (1994) The Ets transcription factors encoded by the Drosophila gene pointed direct glial differentiation in the embryonic CNS. Cell 78:149-160.

Klämbt C (1993) The Drosophila gene pointed encodes two ETS-like proteins which are involved in the development of the midline glial cells. Development 117:163-176.

Klämbt C, Glazer L, Shilo B-Z (1992) breathless, a Drosophila FGF receptor homolog, is essential for migration of tracheal and specific midline glial cells. Genes Dev 6:1668-1678.

Lanoue BR, Gordon MD, Battye R, Jacobs JR (2000) Genetic analysis of vein function in the Drosophila embryonic nervous system. Genome 43:564-573.

Lee T, Luo L (1999) Mosaic analysis with a repressible cell marker for studies of gene function in neuronal morphogenesis. Neuron 22:451-461.

Lee T, Feig L, Montell DJ (1996) Two distinct roles for Ras in a developmentally regulated cell migration. Development 122:409-418.

Lu X, Chou T-B, Williams NG, Roberts T, Perrimon N (1993) Control of cell fate determination by $\mathrm{p} 21^{\mathrm{ras}} / \mathrm{Ras} 1$, an essential component of torso signaling in Drosophila. Genes Dev 7:621-632.

Manning L, Doe CQ (1999) Prospero distinguishes sibling cell fate without asymmetric localization in the Drosophila adult external sense organ lineage. Development 126:2063-2071.

Nüsslein-Volhard C, Wieschaus E, Kluding H (1984) Mutations affecting the pattern of the larval cuticle in Drosophila melanogaster. I. Zygotic loci on the second chromosome. Roux's Arch Dev Biol 193:267-282. 
Okabe M, Okano H (1997) Two-step induction of chordotonal organ precursors in Drosophila embryogenesis. Development 124:1045-1053.

O’Keefe L, Dougan ST, Gabay L, Raz E, Shilo BZ, DiNardo S (1997) Spitz and Wingless, emanating from distinct borders, cooperate to establish cell fate across the Engrailed domain in the Drosophila epidermis. Development 124:4837-4845.

Perrimon N, Perkins LA (1997) There must be 50 ways to rule the signal: the case of the EGF receptor. Cell 89:13-16.

Pielage J, Klämbt C (2001) Glial cells aid target selection. Trends Neurosci 24:432-433.

Queenan AM, Ghabrial A, Schupbach T (1997) Ectopic activation of torpedo/Egfr, a Drosophila receptor tyrosine kinase, dorsalizes both the eggshell and the embryo. Development 124:3871-3880.

Rangarajan R, Courvoisier H, Gaul U (2001) Dpp and Hedgehog mediate neuron-glia interactions in Drosophila eye development by promoting the proliferation and motility of subretinal glia. Mech Dev 108:93-103.

Rebay I, Rubin GM (1995) Yan functions as a general inhibitor of differentiation and is negatively regulated by activation of the Ras1/MAPK pathway. Cell 81:857-866.

Reddy GV, Rodrigues V (1999a) Sibling cell fate in the Drosophila adult external sense organ lineage is specified by Prospero function which is regulated by Numb and Notch. Development 126:2083-2092.

Reddy GV, Rodrigues V (1999b) A glial cell arises from an additional division within the mechanosensory lineage during development of the microchaete on the Drosophila notum. Development 126:4617-4622.

Reich A, Shilo B-Z (2002) Keren, a new ligand of the Drosophila epidermal growth factor receptor, undergoes two modes of cleavage. EMBO J 21:4287-4296.

Rusten TE, Cantera R, Urban J, Technau G, Kafatos FC, Barrio R (2001) Spalt modifies EGFR-mediated induction of chordotonal precursors in the embryonic PNS of Drosophila promoting the development of oenocytes. Development 128:711-722.

Rutledge BJ, Zhang K, Bier E, Jan YN, Perrimon N (1992) The Drosophila spitz gene encodes a putative EGF-like growth factor involved in dorsalventral axis formation and neurogenesis. Genes Dev 6:1503-1517.
Schejter ED, Shilo B-Z (1989) The Drosophila EGF receptor homolog (DER) gene is allelic to faint little ball, a locus essential for embryonic development. Cell 56:1093-1104.

Scholz H, Sadlowski E, Klaes A, Klämbt C (1993) Genetic dissection of pointed, a Drosophila gene encoding two ETS-related proteins. Genetics 135:455-468

Scholz H, Deatrick J, Klaes A, Klämbt C (1997) Control of midline glia development in the embryonic Drosophila CNS. Mech Dev 64:137-151.

Sepp KJ, Auld VJ (2003) RhoA and Racl GTPases mediate the dynamic rearrangement of actin in peripheral glia. Development 130:1825-1835.

Sepp KJ, Schulte J, Auld VJ (2000) Developmental dynamics of peripheral glia in Drosophila melanogaster. Glia 30:122-133.

Sepp KJ, Schulte J, Auld VJ (2001) Peripheral glia direct axon guidance across the CNS/PNS transition zone. Dev Biol 238:47-63.

Settleman J (2001) Rac 'n Rho: the music that shapes a developing embryo. Dev Cell 1:321-331.

Shishido E, Ono N, Kojima T, Saigo K (1997) Requirements of DFR1/ Heartless, a mesoderm-specific Drosophila FGF-receptor, for the formation of heart, visceral and somatic muscles, and ensheathing of longitudinal axon tracts in CNS. Development 124:2119-2128.

Simcox AA, Grumbling G, Schnepp B, Bennington-Mathias C, Hersperger E, Shearn A (1996) Molecular, phenotypic, and expression analysis of vein, a gene required for growth of the Drosophila wing disc. Dev Biol 177:475-489.

Sonnenfeld MJ, Jacobs JR (1994) Mesectodermal cell fate analysis in Drosophila midline mutants. Mech Dev 46:3-13.

Sonnenfeld MJ, Jacobs JR (1995) Apoptosis of the midline glia during Drosophila embryogenesis: a correlation with axon contact. Development 121:569-578.

Verkhusha VV, Tsukita S, Oda H (1999) Actin dynamics of lamellipodia in the Drosophila ovary revealed by a GFP-actin fusion protein. FEBS Lett 445:395-401.

Wing JP, Schwartz LM, Nambu JR (2001) The RHG motifs of Drosophila Reaper and Grim are important for their distinct cell death-inducing abilities. Mech Dev 102:193-203. 